\title{
Research Article \\ Optimal Sliding Mode Controllers for Attitude Stabilization of Flexible Spacecraft
}

\author{
Chutiphon Pukdeboon \\ Department of Mathematics, Faculty of Applied Science, King Mongkut's University of \\ Technology North Bangkok, Bangkok 10800, Thailand \\ Correspondence should be addressed to Chutiphon Pukdeboon, cpd@kmutnb.ac.th
}

Received 4 October 2010; Revised 16 May 2011; Accepted 16 May 2011

Academic Editor: Alexander P. Seyranian

Copyright (C) 2011 Chutiphon Pukdeboon. This is an open access article distributed under the Creative Commons Attribution License, which permits unrestricted use, distribution, and reproduction in any medium, provided the original work is properly cited.

\begin{abstract}
The robust optimal attitude control problem for a flexible spacecraft is considered. Two optimal sliding mode control laws that ensure the exponential convergence of the attitude control system are developed. Integral sliding mode control (ISMC) is applied to combine the first-order sliding mode with optimal control and is used to control quaternion-based spacecraft attitude manoeuvres with external disturbances and an uncertainty inertia matrix. For the optimal control part the state-dependent Riccati equation (SDRE) and optimal Lyapunov techniques are employed to solve the infinite-time nonlinear optimal control problem. The second method of Lyapunov is used to guarantee the stability of the attitude control system under the action of the proposed control laws. An example of multiaxial attitude manoeuvres is presented and simulation results are included to verify the usefulness of the developed controllers.
\end{abstract}

\section{Introduction}

In recent years considerable attention has been focused on the optimal control problems of a spacecraft. Various optimal control methods have been proposed for solving the attitude control problems of a rigid spacecraft. Nonlinear $H_{\infty}$ control was used in [1] to design a stabilizing feedback control for the spacecraft tracking problem. Sharma and Tewari [2] devised a Hamilton-Jacobi formulation for tracking attitude manoeuvres of spacecraft to derive a nonlinear optimal control law. An $H_{\infty}$ inverse optimal adaptive controller was applied to attitude tracking of spacecraft by Luo et al. [3]. An adaptive control and nonlinear $H_{\infty}$ control were merged to design robust optimal controllers. An alternative way to design a robust optimal controller is to use an optimal sliding mode controller design scheme. Sliding mode control (SMC) is a very effective approach when applied to a system with disturbances which satisfy the matched uncertainty condition. In recent decade, SMC has widely been extended to incorporate new techniques, such as adaptive sliding mode control, higher-order 
sliding mode control, and optimal sliding mode control. These techniques preserve the main advantages of SMC and also yield more accuracy and desired performances [4,5]. Various real life applications have been controlled by practical implementations of these advanced SMC techniques. Adaptive sliding mode controllers were used to control manipulators $[6,7]$ and a hypersonic flight vehicle [8]. Second-order sliding mode controllers have been applied to electrical devices (container cranes [9], DC drives [10,11]), practical mechanical systems [12], aircraft pitch control [13], and spacecraft attitude tracking control [14]. On the other hand, optimal sliding mode control has been rarely studied for practical applications since this method requires the knowledge of a stabilizing control law to solve a nonlinear optimal control problem. Optimal sliding mode control has been developed by $\mathrm{Xu}$ [15] by employing the ISMC concept [16] to combine the first-order sliding mode with optimal control. Unfortunately, the class of nonlinear systems studied in [15] is a special case of nonlinear systems and cannot be applied to more general highly nonlinear systems (e.g., spacecraft system). The $\mathrm{Xu}$ method [15] has further been extended to a more general class of nonlinear systems in [17]. Pukdeboon and Zinober [17] have developed robust optimal control laws for attitude tracking of a rigid spacecraft. However, the optimal sliding mode control of a flexible spacecraft has rarely been studied. An optimal sliding mode controller was developed in [18] for a linear stochastic system and applied to the middeck active control experiment (MACE) which represents the control structure interaction problem for a precision spacecraft. The integrated controller combining the optimal sliding mode and active vibration suppression control was presented in $\mathrm{Hu}$ and $\mathrm{Ma}$ [19]. For their control strategy, the technique of active vibration control using smart materials was used to actively suppress certain flexible modes by designing optimal positive position feedback (OPPF) compensators that add damping to the flexible structures in certain critical modes in the inner feedback loop.

In this paper the SDRE approach is used for nonlinear optimal controller designs, The SDRE approach was applied to optimal control and stabilization for nonlinear systems by Banks and Mhana [20]. The explicit control law has been studied for nonlinear system of the form $\dot{x}=A(x) x+B(x) u$. In [21] Cloutier et al. studied nonlinear regulation and nonlinear $H_{\infty}$ control via the SDRE approach. Some real-life applications have been successfully controlled by implementation of the SDRE technique. In [22, 23] the SDRE method was successfully applied to spacecraft attitude control for rest-to-rest manoeuvres. Zhang et al. [24] has applied the SDRE technique to propose optimal controllers for a flexible transporter system with arbitrarily varying lengths. On the other hand, a nonlinear optimal controller can be developed by using the optimality Hamilton-Jacobi-Bellman principle. This concept was used to design suboptimal control laws for the Euler-Lagrange systems in [25-27]. One approach to solve the equations obtained by the optimality Hamilton-Jacobi-Bellman principle is to use the optimal Lyapunov technique and its corresponding theorems. Based on Krasovskii's theorem and the optimal Lyapunov technique, many optimal controllers have been developed for attitude stabilization of a rigid body by El-Gohary and coresearchers [28-31].

The class of nonlinear systems studied in [15] was a special case of nonlinear systems whereas our design will work for a more general class of nonlinear systems. We have developed two controllers for application to spacecraft regulation manoeuvres. The first controller is an SDRE-based sliding mode (SM) controller. It uses the method in [15] and combines this with ISMC [16] and the SDRE approach [20]. Since spacecraft systems are highly nonlinear systems, the SDRE approach is rather difficult to apply for spacecraft systems. The basic concepts in [32] are used for applying the SDRE approach to the spacecraft system. For the second controller, the optimal Lyapunov technique [28-31] is applied to 
develop an optimal control law and the ISMC concept is used to merge the resulting optimal controller and first-order sliding control. The control law obtained could be called an optimal Lyapunov-based SM controller.

This paper is organized as follows. In Section 2 the dynamic equations of a fexible spacecraft and the attitude kinematics [33,34] are described. Section 3 presents the integral sliding mode [16] technique. In Section 4 we discuss an optimal sliding mode controller and study the sliding manifold and control law in the stability proof of this controller. Section 5 describes the SDRE technique and includes a systematic procedure for the design of the SDRE controller. In Section 6 the optimal Lyapunov technique is used to produce an optimal feedback controller that yields global asymptotic stability. A controller design procedure is proposed and the stability proof of this controller is investigated. In Section 7 an example of spacecraft attitude manoeuvres is presented in order to make comparisons between the SDRE-based SM controller and the optimal Lyapunov-based SM controller. In Section 8 we present conclusions.

\section{Mathematical Model of Flexible Spacecraft}

A flexible spacecraft is composed of a rigid main body and some flexible appendages. The kinematics of the spacecraft determine the attitude of the main body and are described by the four unitary quaternions [34]

$$
q=e \sin \left(\frac{\beta}{2}\right), \quad \cos \left(\frac{\beta}{2}\right)
$$

where $e \in R^{3}$ and $\beta$ denote the Euler axis and Euler angle, respectively. We define here the quaternion $\bar{q}=\left[\begin{array}{ll}q_{0} & q^{T}\end{array}\right]^{T}$ with $q \in R^{3}$. Then the kinematic equations are described in terms of the attitude quaternion [34] and are given by

$$
\dot{\bar{q}}=\frac{1}{2}\left[\begin{array}{c}
-q^{T} \\
{[q \times]+q_{0} I_{3 \times 3}}
\end{array}\right] \omega
$$

where $\omega \in R^{3}$ denotes the angular velocity vector and $I_{3 \times 3}$ is the $3 \times 3$ identity matrix. The skew-symmetric matrix $[q \times]$ is

$$
[q \times]=\left[\begin{array}{ccc}
0 & -q_{3} & q_{2} \\
q_{3} & 0 & -q_{1} \\
-q_{2} & q_{1} & 0
\end{array}\right]
$$

and the elements of $\bar{q}$ are restricted by $\|\bar{q}\|=1$. Note that a quaternion consists of the scalar $q_{0}$ and the three-dimensional vector $q$, so it has four components. The scalar term is used for avoidance of singular points in the attitude representation [34]. The quaternion kinematics equation is required to be solved for all four components. However, to indicate the orientation of the spacecraft or a rotational motion, it is sufficient to use only the vector $q$ because this vector completely shows rotation axis and angle. Furthermore, the scalar $q_{0}$ can be calculated 
easily using the vector $q$ and the condition $\|\bar{q}\|=1$. More details of quaternion and other attitude representations can be found in $[34,35]$.

The equation governing a flexible spacecraft is expressed as [33]

$$
\begin{gathered}
J \dot{\omega}+\delta^{T} \ddot{\eta}=-[\omega \times]\left(J \omega+\delta^{T} \dot{\eta}\right)+u+d, \\
\ddot{\eta}+C \dot{\eta}+K \eta=-\delta_{1} u_{p}-\delta J \dot{\omega},
\end{gathered}
$$

where $J=J^{T}$ is the total inertia matrix of the spacecraft, $\eta$ is the modal displacement, and $\delta$ is the coupling matrix between the central rigid body and the flexible attachments. $u \in \mathcal{R}^{3}$ denotes the control input, $d \in \mathcal{R}^{3}$ represents the external disturbance torque, and $K$ and $C$ denote the stiffness and damping matrices, respectively, which are defined as

$$
\begin{gathered}
K=\operatorname{diag}\left(\omega_{n i}^{2}, i=1,2, \ldots, N\right), \\
C=\operatorname{diag}\left(2 \zeta_{i} \omega_{n i}, i=1,2, \ldots, N\right)
\end{gathered}
$$

with damping $\zeta_{i}$ and natural frequency $\omega_{n i}$.

Letting

$$
\vartheta=\left[\begin{array}{c}
\eta \\
\dot{\eta}+\delta \omega
\end{array}\right]
$$

the relative dynamic equation (2.4) can be written as

$$
\begin{gathered}
J_{m b} \dot{\omega}=-[\omega \times]\left(J_{m b} \omega+H \vartheta\right)+L \vartheta-M \omega+u+d, \\
\dot{\vartheta}=A \vartheta+B \omega,
\end{gathered}
$$

where

$$
\begin{gathered}
A=\left[\begin{array}{cc}
0 & I \\
-K & -C
\end{array}\right], \quad B=\left[\begin{array}{c}
-\delta \\
C \delta
\end{array}\right], \quad D=\left[\begin{array}{c}
0 \\
-\delta
\end{array}\right], \\
H=\left[\begin{array}{ll}
0 & \delta^{T}
\end{array}\right], \quad L=\left[\begin{array}{ll}
\delta^{T} K & \delta^{T} C
\end{array}\right], \quad M=\delta^{T} C \delta .
\end{gathered}
$$

Note that because of the constraint relation among the four unitary quaternions $q_{0}^{2}+$ $q^{T} q=1$, and $q_{0}$ are not independent of the other components of the quaternion.

\section{ISMC}

The concept of integral sliding mode [16] concentrates on the robustness of the motion in the whole state space. It is constructed without the reaching phase and it ensures insensitivity of the desired trajectory with respect to matched uncertainties, starting at time zero. However, since this controller design requires a first-order SMC, the main drawback of sliding control, namely, the chatter problem, is encountered. 
Integral sliding control is applied to obtain robustness against external disturbances during the sliding mode. Next the concepts of ISMC [16] are discussed.

Consider a nonlinear dynamic system

$$
\dot{x}=F(x)+G(x) u,
$$

where $x \in \mathcal{R}^{n}, u \in \mathcal{R}^{m}$. Suppose there exists a feedback control law $u=u_{0}(x)$ such that system (3.1) can be stabilized to follow a desired trajectory.

For system (3.1) the control law [16] is designed as

$$
u=u_{0}+u_{1}
$$

where $u_{0}$ is the ideal control and $u_{1}$ is designed to be discontinuous to reject the disturbances. The switching function [16] is designed as

$$
s=s_{0}(x)+\gamma, \quad \text { with } s, s_{0}(x), \gamma \in \mathcal{R}^{m} \text {. }
$$

The switching function consists of two parts; the first part $s_{0}(x)$ may be designed as the linear combination of the system states (similar to the conventional sliding mode design); and the second part $\gamma$ induces the integral term and will be determined as

$$
\dot{\gamma}=-\frac{\partial s_{0}}{\partial x}\left(F(x)+G(x) u_{0}(x)\right), \quad r(0)=-s_{0}(x(0))
$$

where $\gamma(0)$ is determined based on the requirement $s(0)=0$ since the sliding mode occurs at time zero. To derive the sliding mode equation, the time derivative of $s$ on the system trajectories should be made equal to zero. The equivalent control $u_{\mathrm{eq}}$ is determined by solving the algebraic equation $\dot{s}=0$ with respect to the control input and then substituting into the equation for $u$.

\section{Optimal Sliding Mode Controller Design}

We continue the controller design by using the ISMC technique. The way to obtain the optimal performance is to design an ideal control in (3.2) using nonlinear optimal control techniques. So our designs will consider an ideal control as the optimal control law for nonlinear attitude regulation systems. (In this paper the SDRE and the optimal Lyapunov approaches are used to produce optimal control laws and the details will be given in subsequent sections.).

We use the integral sliding mode concept to obtain the sliding manifold and a new robust optimal control law is then developed. The second method of Lyapunov is used to show that reaching and sliding on the manifold are guaranteed.

Using (3.3) and letting $s_{0}(x)=\omega+\kappa q$, the switching function is designed as

$$
s=\omega+\kappa q+\phi,
$$


where $\kappa$ is a $3 \times 3$ symmetric positive-definite constant matrix and $\phi$ is an auxiliary variable that is the solution of the differential equation [16]

$$
\dot{\phi}=-\frac{\partial s_{0}}{\partial x}\left[F(x)+G(x) v^{*}\right], \quad \phi(0)=-s_{0}(x(0))
$$

with $v^{*}$ being the optimal controller for the system (3.1). Using the ISMC (3.2) we obtain an optimal sliding mode controller

$$
u=v^{*}-\mu \tau
$$

where $\mu$ is a $m \times m$ positive definite diagonal matrix, and $\tau \in \mathcal{R}^{m}$ the $i$ th component of $\tau$ is given by

$$
\begin{gathered}
\tau_{i}=\operatorname{sat}\left(s_{i}, \varepsilon_{i}\right), \quad i=1,2,3, \ldots m \\
\operatorname{sat}\left(s_{i}, \varepsilon_{i}\right)= \begin{cases}1 & \text { for } s_{i}>\varepsilon_{i} \\
s_{i} / \varepsilon_{i} & \text { for }\left|s_{i}\right| \leq \varepsilon_{i} \\
-1 & \text { for } s_{i}<-\varepsilon_{i}\end{cases}
\end{gathered}
$$

Now we show that the control law (4.3) is designed such that the reaching and sliding mode conditions are satisfied. The candidate Lyapunov is selected as [18]

$$
V_{s}=\frac{1}{2} s^{T} S
$$

and the time derivative of $V$ is

$$
\dot{V}_{S}=s^{T} \dot{s}
$$

With the substitution of $\dot{s}$ and (4.2) we obtain

$$
\dot{V}_{s}=s^{T}\left(\frac{\partial s_{0}}{\partial x}\left[F(x)+G(x) v^{*}\right]+\dot{\phi}\right) .
$$

With external disturbances and the optimal control $v^{*}$, the control law (3.2) can be written as

$$
u=u_{1}+v^{*}+\xi
$$


Substituting (4.2) and (4.8) in (4.7), the time derivative of $V_{s}$ can be written as

$$
\begin{aligned}
\dot{V}_{s}= & s^{T} \frac{\partial s_{0}}{\partial x}(F(x)+G(x) u) \\
& -s^{T}\left[\frac{\partial s_{0}}{\partial x}\left(F(x)+G(x) u-G(x) u_{1}-G(x) \xi\right)\right] \\
= & s^{T}\left(\frac{\partial s_{0}}{\partial x} G(x)\left[\xi+u_{1}\right]\right) .
\end{aligned}
$$

Let the discontinuous control input $u_{1}$ have the following form

$$
u_{1}=-\mu \operatorname{sign}(s)
$$

where $\mu \in \mathcal{R}^{m \times m}$ is a positive definite diagonal matrix. Letting $\Psi=\left(\partial s_{0} / \partial x\right) G(x)$, we obtain

$$
\dot{V}_{s}=s^{T}(\Psi[\xi-\mu \operatorname{sign}(s)]) .
$$

We choose $s_{0}$ such that $\Psi$ is positive definite and then (4.11) becomes

$$
\dot{V}_{s}=|s|(\Psi[\xi \operatorname{sign}(s)-\mu]) .
$$

Obviously if $\mu$ is chosen such that $\mu>\sup |\xi|$, then $\dot{V}_{s}<0$. This guarantees reaching and sliding on the manifold.

\section{SDRE Controller}

In this section we mention briefly the optimal controller design scheme using the SDRE approach. The regulation motion of a flexible spacecraft is considered. The Xu method [15] and the integral sliding mode [16] are merged to design a new controller which consists of two parts; the sliding mode and optimal control. The first-order sliding mode is used for the sliding mode controller design while the optimal control law is designed using the SDRE method [20] to solve the infinite-time optimal quadratic problem.

The SDRE method requires factorization of the nonlinear dynamics into the state vector and the product of a matrix valued function which depends the state itself. This matrix is so called the state-dependent coefficient (SDC) matrix. For the optimal controller design, the difficulty of using the SDRE approach is how choose the appropriate SDC matrix. Using the basic concepts described in [32] we can rewrite the spacecraft dynamics equation in a more suitable form and the appropriate SDC matrix is then selected. After we obtain the optimal control law, a new optimal sliding mode controller will be designed by merging the optimal control and first-order sliding mode controller.

This controller is designed such that it minimizes the performance index

$$
I=\int_{0}^{\infty}\left(x^{T} Q(x) x+u^{T} N(x) x+u^{T} R(x) u\right) d t
$$


where

$$
\begin{gathered}
\dot{x}=f(x)+g(x) u, \quad x(0)=x_{0}, \\
f(x)=\left[\begin{array}{c}
-J_{m b}^{-1}\left([\omega \times] J_{m b} \omega+[\omega \times] H \vartheta+M \omega-L \vartheta\right) \\
0.5\left([q \times]+q_{0} I_{3}\right) \\
A \vartheta+B \omega
\end{array}\right], \quad g(x)=\left[\begin{array}{c}
J_{m b}^{-1} \\
0_{3 \times 3} \\
0_{3 \times 3}
\end{array}\right] .
\end{gathered}
$$

Here, the state $x \in \mathcal{R}^{n}$ is defined as $x=\left[\begin{array}{lll}\omega^{T} & q^{T} \vartheta^{T}\end{array}\right]^{T}$. The weighting matrices $Q(x), N(x)$, and $R(x)$ are positive semidefinite and positive definite, respectively. $f(x)$ and $g(x)$ are all sufficiently smooth functions of the state vector $x(t)$, and $x(0)$ is the initial conditions of the process. It is assumed that $f(0)=0$ and $g(x) \neq 0$ for all $x$.

To apply the SDRE method $f(x)$ must be decomposed as $f(x)=a(x) x$. Using the theorem in [32] the SDC matrix $a(x)$ can be obtained by writing $f(x)$ in a more suitable form as

$$
f(x)=\left[\begin{array}{ccc}
-J_{m b}^{-1}[\omega \times] J_{m b}-J_{m b}^{-1} M & 0_{3 \times 3} & -J_{m b}^{-1}[\omega \times] H+J_{m b}^{-1} L \\
0.5\left([q \times]+q_{0} I_{3}\right) & 0_{3 \times 3} & 0_{3 \times 8} \\
B & 0_{8 \times 3} & A
\end{array}\right]\left[\begin{array}{c}
\omega \\
q \\
\vartheta
\end{array}\right] .
$$

To use the SDRE approach the SDC matrix $a(x)$ is chosen as

$$
a(x)=\left[\begin{array}{ccc}
-J_{m b}^{-1}[\omega \times] J_{m b}-J_{m b}^{-1} M & 0_{3 \times 3} & -J_{m b}^{-1}[\omega \times] H+J_{m b}^{-1} L \\
0.5\left([q \times]+q_{0} I_{3}\right) & 0_{3 \times 3} & 0_{3 \times 8} \\
B & 0_{8 \times 3} & A
\end{array}\right] .
$$

Thus, the optimal control $v^{*}[15]$ is given as

$$
v^{*}=-R^{-1}\left(g^{T} \Pi(x)+N(x)\right) x,
$$

where $\Pi(x)$ is the solution to the generalized SDRE [15]

$$
\begin{aligned}
\Pi(x) & {\left[a(x)-g(x) R^{-1}(x) N^{T}(x)\right]+\left[a^{T}(x)-N(x) R^{-1}(x) g^{T}(x)\right] \Pi(x)+Q(x) } \\
& -\Pi(x) g(x) R^{-1}(x) g^{T}(x) \Pi(x)-N(x) R^{-1} N^{T}(x)=0_{14 \times 14} .
\end{aligned}
$$

Note that the parameterization in (5.3) is not unique. It has explicitly been selected to make the stability problem analytically tractable. It is convenient to use this parameterization, since the state-dependent Riccati equation (5.6) will have a simple form to be solved for $\Pi(x)$. Next, 
it should be checked whether the parametrization in (5.3) is pointwise controllable for all $x$. The controllability matrix is

$$
\begin{aligned}
M_{C} & =\left[\begin{array}{lll}
g(x) & a(x) g(x) & a^{2}(x) g(x)
\end{array}\right] \\
& =\left[\begin{array}{ccc}
J_{m b}^{-1} & -J_{m b}^{-1}[\omega \times]-J_{m b}^{-1} M J_{m b}^{-1} & M_{C}^{13} \\
0_{3 \times 3} & 0.5\left([q \times]+q_{0} I_{3}\right) J_{m b}^{-1} & M_{C}^{23} \\
0_{3 \times 3} & B J_{m b}^{-1} & M_{C}^{33}
\end{array}\right],
\end{aligned}
$$

where

$$
\begin{gathered}
M_{C}^{13}=\left(J_{m b}^{-1}[\omega \times]+J_{m b}^{-1} M J_{m b}^{-1}\right)\left([\omega \times]-M J_{m b}^{-1}\right)+\left(-J_{m b}^{-1}[\omega \times] H+J_{m b}^{-1} L\right) B J_{m b}^{-1}, \\
M_{C}^{23}=0.5\left([q \times]+q_{0} I_{3}\right)\left(-J_{m b}^{-1}[\omega \times]-J_{m b}^{-1} M J_{m b}^{-1}\right), \\
M_{C}^{33}=B\left(-J_{m b}^{-1}[\omega \times]-J_{m b}^{-1} M J_{m b}^{-1}\right)+A B J_{m b}^{-1} .
\end{gathered}
$$

Clearly the condition for pointwise controllability is

$$
\left|M_{C}\right|=\left|J_{m b}^{-1}\right| \begin{array}{cc}
0.5\left([q \times]+q_{0} I_{3}\right) J_{m b}^{-1} & M_{C}^{23} \\
B J_{m b}^{-1} & M_{C}^{33}
\end{array} \mid,
$$

where $\left|M_{C}\right|$ denotes the determinant of the matrix $M_{C}$. Letting $T(\bar{q})=[q \times]+q_{0} I_{3}$, one obtains

$$
\left|M_{c}\right|=\left|J_{m b}^{-1}\right|\left|\frac{1}{2} T(\bar{q}) J_{m b}^{-1} A B J_{m b}^{-1}\right|
$$

Using (5.10) we can ensure that $\left|M_{C}\right| \neq 0$. This implies that $M_{C}$ has full rank. In Cimen [36] it was stated that the observability matrix has full rank by choosing the $Q(x)$ positive definite for all $x \in R^{n}$. If our design selects $Q(x)$ to be positive definite, the observability matrix will be a full rank matrix and the sufficient condition for observability is satisfied. Thus, the SDRE method generates a closed loop solution, which is locally asymptotically stable. However, the success of the SDRE approach depends on a good choice of the matrix $a(x)$. It is difficult to obtain global stability because of the limitations of this technique.

After we obtain the matrix $a(x)$, an optimal controller $v^{*}$ can be designed using (5.5). Substituting this control into (4.3) the SDRE-based SM controller is obtained.

\section{Optimal Lyapunov Controller}

This section presents an optimal control moment which stabilizes the zero solution and minimizes the selected performance index (5.1). The basic principles in Krasovskii [37] with a Lyapunov function are applied to develop an optimal controller. The stability proof of this control law is also performed by using the optimal Lyapunov technique (see [28-31]). 
The optimal Lyapunov function, defined as the minimum value of the performance index (5.1), is given by

$$
V(x)=\min _{u} \frac{1}{2} \int_{0}^{\infty}\left(x^{T} Q(x) x+2 u^{T} N(x) x+u^{T} R(x) u\right) d t .
$$

The function $V(x)$ represents the value of the integral performance index when evaluated along the optimal trajectory. Let the positive definite symmetric matrix $Q \in \mathcal{R}^{n \times n}$ and the matrix $N \in \mathcal{R}^{m \times n}$ be partitioned as

$$
Q=\left[\begin{array}{lll}
Q_{11} & Q_{12} & Q_{13} \\
Q_{21} & Q_{22} & Q_{23} \\
Q_{31} & Q_{32} & Q_{33}
\end{array}\right], \quad N=\left[\begin{array}{lll}
N_{1} & N_{2} & N_{3}
\end{array}\right]
$$

respectively. We next show that $V(x)$ can be treated as a Lyapunov function. Based on Krasovskii's theorem, the function $V(x)$ satisfies the following equation:

$$
B(V, x, u)=\frac{\partial V}{\partial t}+\left(\nabla_{\omega} V\right) \dot{\omega}+\left(\nabla_{\bar{q}} V\right) \dot{\bar{q}}+\left(\nabla_{\vartheta} V\right) \dot{\vartheta}+\Omega(V, x, u) \geq 0
$$

We assume that $u_{o}$ is the optimal control law. From the optimality conditions of Krasovskii's theorem, function $V$ must satisfy the following partial differential equation:

$$
0=\frac{\partial V}{\partial t}+\frac{\partial V}{\partial \omega} \dot{w}+\frac{\partial V}{\partial \bar{q}} \dot{\bar{q}}+\frac{\partial V}{\partial \vartheta} \dot{\vartheta}+\frac{1}{2}\left(x^{T} Q x+2 u_{u_{o}}^{T} N x+u_{u_{o}}^{T} R u_{u_{o}}\right)
$$

which is known as the Hamilton-Jacobi equation. Clearly, the resulting partial differential equation (6.4) contains the optimal control $u_{o}$. In particular if the optimal control $u_{o}$ is known, we can solve this equation for the optimal Lyapunov function $V$. On the other hand if the function $V$ is known, this equation can be solved for the optimal control $u_{0}$. In fact both solutions of this equation and control torques are dependent on each other. Substituting (2.2) and (2.7) into (6.4), one obtains

$$
\begin{aligned}
0= & \frac{\partial V}{\partial t}+\frac{\partial V}{\partial \omega}\left(J_{m b}^{-1}\left(-[\omega \times] J_{m b} \omega-[\omega \times] H \vartheta-M \omega+L \vartheta+u_{o}\right)\right) \\
& +\frac{\partial V}{\partial \bar{q}}\left[-\frac{1}{2}\left(q^{T} \omega\right)^{T} \frac{1}{2}(T(\bar{q}) \omega)^{T}\right]^{T}+\frac{\partial V}{\partial \vartheta}(A \vartheta+B \omega) \\
& +\frac{1}{2} \omega^{T} Q_{11} \omega+\frac{1}{2} q^{T} Q_{22} q+\frac{1}{2} \vartheta^{T} Q_{33} \vartheta+\omega^{T} Q_{12} q+\omega^{T} Q_{13} \vartheta+q^{T} Q_{23} \vartheta \\
& +u_{o}^{T} N\left[\begin{array}{lll}
\omega^{T} & q^{T} & \left.\vartheta^{T}\right]+\frac{1}{2} u_{o}^{T} R u_{o} .
\end{array}\right.
\end{aligned}
$$


Let a Lyapunov function be chosen as

$$
V(x)=\frac{1}{2} \omega^{T} J_{m b} \omega+\alpha\left(q_{0}-1\right)^{2}+\alpha q^{T} q+\frac{1}{2} \vartheta^{T} P \vartheta
$$

where $P$ is a positive-definite matrix that is a solution of the Lyapunov equation $A^{T} P+P A=$ $-Q_{A}$ with a positive-definite matrix $Q_{A}$. The gradient of $V$ with respect to $\omega, \bar{q}$ and $\vartheta$ is given by

$$
\frac{\partial V}{\partial \omega}=\omega^{T} J_{m b}, \quad \frac{\partial V}{\partial \bar{q}}=\left[\begin{array}{cc}
2 \alpha\left(q_{0}-1\right) & 2 \alpha q
\end{array}\right], \quad \frac{\partial V}{\partial \vartheta}=\vartheta^{T} P
$$

This function consists of the sum of quadratic terms, so it is a positive definite function with respect to stabilizing variables. Substituting (6.7) into (6.5), one obtains

$$
\begin{aligned}
0= & \omega^{T} L \vartheta-\omega^{T} M \omega+\omega^{T} u_{o}+\frac{1}{2} \omega^{T} Q_{11} \omega+\frac{1}{2} q^{T} Q_{22} q+\frac{1}{2} \vartheta^{T} Q_{22} \vartheta+\omega^{T} Q_{12} q \\
& +\omega^{T} Q_{13} \vartheta+q^{T} Q_{23} \vartheta+u_{o}^{T} N\left[\begin{array}{ccc}
\omega^{T} & q^{T} & \vartheta^{T}
\end{array}\right]+\frac{1}{2} u_{o}^{T} R u_{o} .
\end{aligned}
$$

After lengthy calculus manipulation, one can obtain the optimal control $u_{o}$ as

$$
u_{o}=-\alpha q-\Lambda \omega
$$

We can conclude that (6.5) can be solved by using the Lyapunov function $V(x)$ and the controller $u_{o}$ with the following relations:

$$
\begin{gathered}
Q_{11}=\Lambda+2 M, \quad Q_{22}=\alpha^{2} \Lambda^{-1}, \quad Q_{33}=Q_{A}=-\frac{1}{2}\left(P A+A^{T} P\right), \\
Q_{31}=Q_{13}^{T}=-\frac{1}{2} P B, \quad Q_{21}=Q_{12}^{T}=0_{3 \times 3}, \quad Q_{32}=Q_{23}^{T}=0_{8 \times 3}, \\
\Lambda=R^{-1}, \quad N_{1}=0_{3 \times 3}, \quad N_{2}=\alpha \Lambda^{-1}, \quad N_{3}=0_{3 \times 8} .
\end{gathered}
$$

We also prove that the optimal control (6.9) with the chosen Lyapunov function (6.6) yields global asymptotic stability. For the purpose of the stability analysis, the first time derivative of $V(x)$ is considered and it takes the form

$$
\dot{V}(x)=\omega^{T} J_{m b} \dot{w}+2 \alpha\left(q_{0}-1\right) \dot{q}_{0}+2 \alpha q^{T} \dot{q}+\vartheta^{T} P \dot{\vartheta}
$$

Substituting (2.7) into (6.11), we obtain

$$
\begin{aligned}
\dot{V}= & \left(\omega^{T} L \vartheta-\omega^{T} M \omega-\alpha \omega^{T} q-\omega^{T} \Lambda \omega\right)+2 \alpha\left(q_{0}-1\right)\left(-\frac{1}{2}\left(q^{T} \omega\right)\right) \\
& +2 \alpha q^{T}\left(\frac{1}{2}\left[q_{0} I_{3}+[q \times]\right]\right) \omega+\vartheta^{T} P(A \vartheta+B \omega)
\end{aligned}
$$




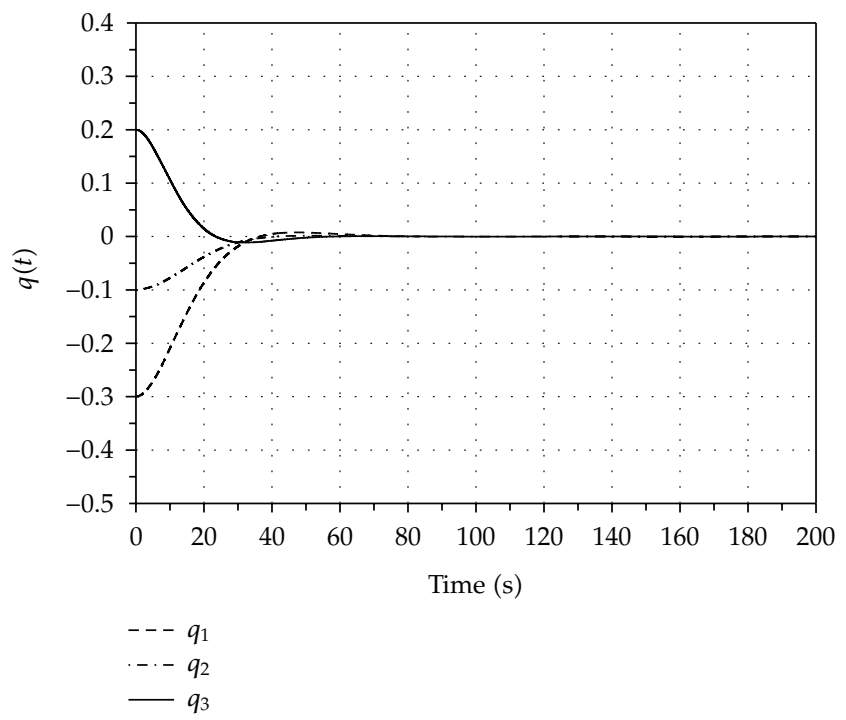

Figure 1: SDRE-based SM controller: components of quaternion vector with external disturbances.

which can be further expressed as

$$
\begin{aligned}
\dot{V}= & \omega^{T} L \vartheta-\omega^{T} M \omega-\alpha \omega^{T} q-\omega^{T} \Lambda \omega \\
& -\alpha q_{0} q^{T} \omega+\alpha q^{T} \omega+\alpha q^{T} q_{0} \omega-\vartheta^{T} Q_{A} \vartheta+\vartheta^{T} P B \omega
\end{aligned}
$$

which can be further expressed as

$$
\dot{V}=-\beta^{T} \Phi \beta,
$$

where

$$
\beta=\left[\begin{array}{l}
\omega \\
\vartheta
\end{array}\right], \quad \Phi=\left[\begin{array}{cc}
\Lambda+M & -L \\
-P B & Q_{A}
\end{array}\right] .
$$

We know that there exists an appropriate controller parameter $\Lambda$ such that $\Phi$ is positive definite. Therefore $\dot{V}(x)$ is negative definite and global asymptotic stability has been proved.

After obtaining the resulting optimal controller, a new optimal sliding mode controller will be developed by combining this controller with a first-order SMC. The optimal Lyapunov-based SM controller can be obtained by letting $v^{*}=u_{0}$ and substituting (6.9) into (4.3).

\section{Simulation Results}

An example of attitude control of flexible spacecraft [33] is presented with numerical simulations to validate and compare both controllers; SDRE-based SM controller and optimal 


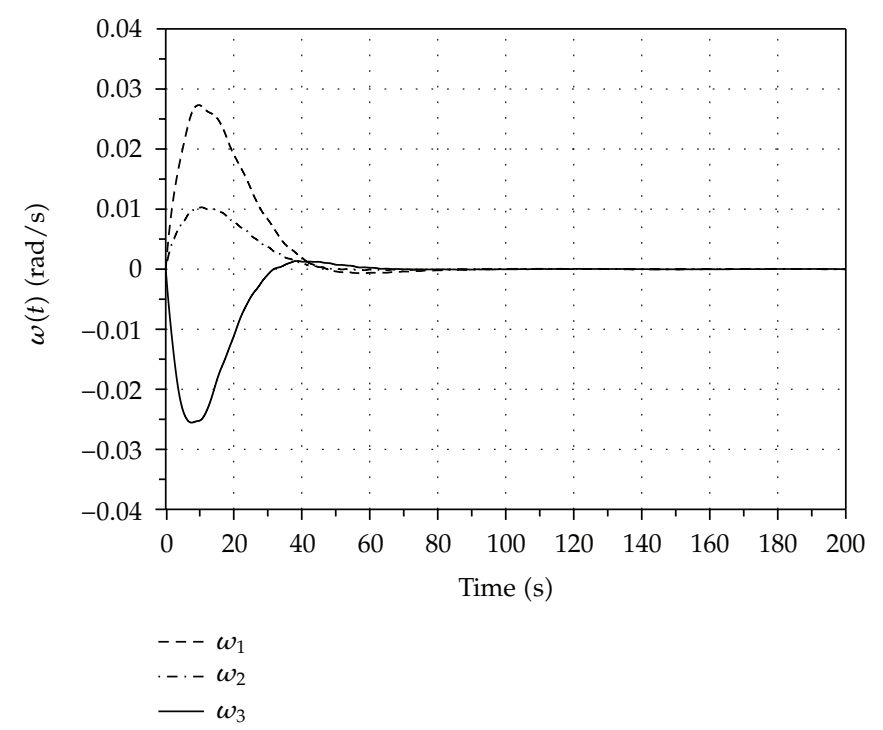

Figure 2: SDRE-based SM controller: components of angular velocity vector with external disturbances.

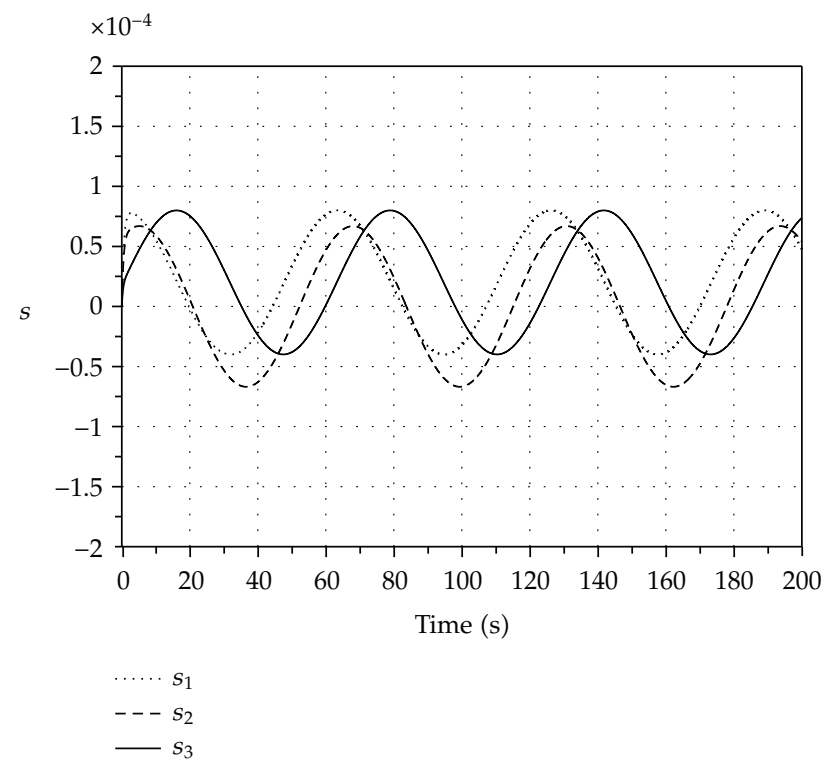

Figure 3: SDRE-based SM controller: switching function with external disturbances.

Lyapunov-based SM controller. The spacecraft is assumed to have the nominal inertia matrix

$$
J=\left[\begin{array}{ccc}
350 & 3 & 4 \\
3 & 270 & 10 \\
4 & 10 & 190
\end{array}\right] \mathrm{kg} \cdot \mathrm{m}^{2}
$$




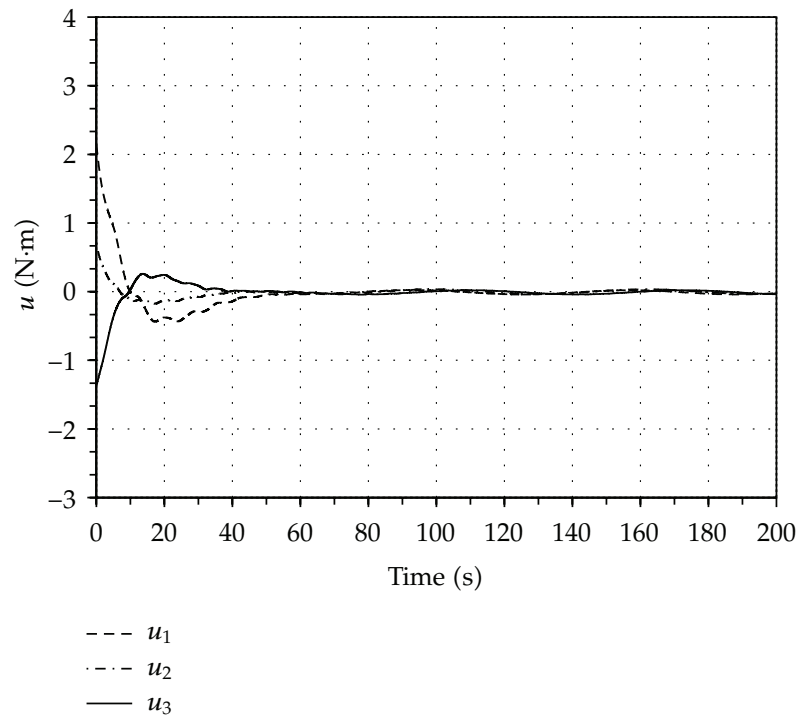

Figure 4: SDRE-based SM controller: control torques with external disturbances.

and coupling matrices

$$
\delta=\left[\begin{array}{ccc}
6.45637 & 1.27814 & 2.15629 \\
-1.25619 & 0.91756 & -1.67264 \\
1.11678 & 2.48901 & -0.83674 \\
1.23637 & -2.6581 & -1.12503
\end{array}\right] \mathrm{kg}^{1 / 2} \cdot \mathrm{m} / \mathrm{s}^{2}
$$

respectively. The first four elastic modes have been considered in the model used for simulating spacecraft at $\omega_{n 1}=0.7681, \omega_{n 2}=1.1038, \omega_{n 3}=1.8733$, and $\omega_{n 4}=2.5496$ with damping $\zeta_{1}=0.0056, \zeta_{2}=0.0086, \zeta_{3}=0.013$, and $\zeta_{4}=0.025$. The weighting matrices are chosen to be $Q=\operatorname{diag}(1,1,1,5,5,5,1,1,1,1,1,1,1,1)$ and $R=\operatorname{diag}(1,1,1)$. The initial states of the rotation motion are given by $\bar{q}(0)=\left[\begin{array}{llll}0.173648 & -0.263201 & 0.789603 & -0.526402\end{array}\right]^{T}, \omega(0)=$ $\left[\begin{array}{lll}0 & 0 & 0\end{array}\right]^{T} \mathrm{rad} / \mathrm{sec}$, and $\vartheta(0)=\left[\begin{array}{llllllll}0 & 0 & 0 & 0 & 0 & 0 & 0 & 0\end{array}\right]^{T}$. For the SDRE-based SM controller the control vector is designed by using (4.3) with optimal control (5.5). The optimal Lyapunov-based SM controller is obtained by using (4.3) with optimal control (6.9). For both controllers the switching function (4.1) is chosen using the same constant matrix $\kappa$ defined as $\kappa=\lambda I_{3 \times 3}$ with $\lambda=1$.2. To obtain $s(0)=0$ the initial $\phi$ is chosen to be $\phi(0)=-(\omega(0)+\kappa q(0))$. The attitude control problem is considered in the presence of external disturbance $d(t)$. The disturbance model [33] is

$$
d(t)=0.1 \times\left[\begin{array}{c}
0.3 \cos \left(\frac{t}{10}\right)+0.1 \\
0.15 \sin \left(\frac{t}{10}\right)+0.3 \cos \left(\frac{t}{10}\right) \\
0.3 \sin \left(\frac{t}{10}\right)+0.1
\end{array}\right] \mathrm{Nm}
$$




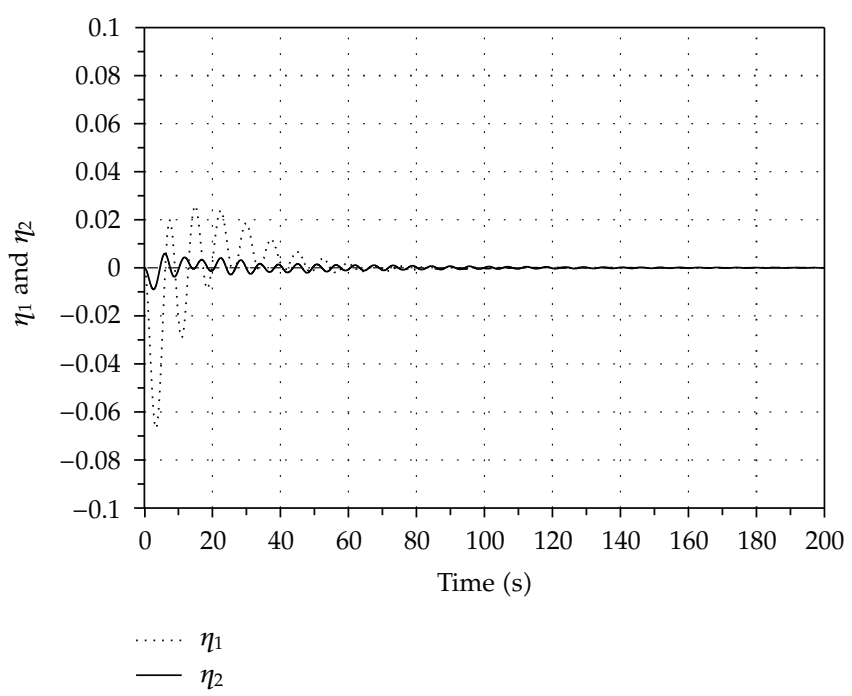

Figure 5: SDRE-based SM controller: modal displacements with external disturbances.

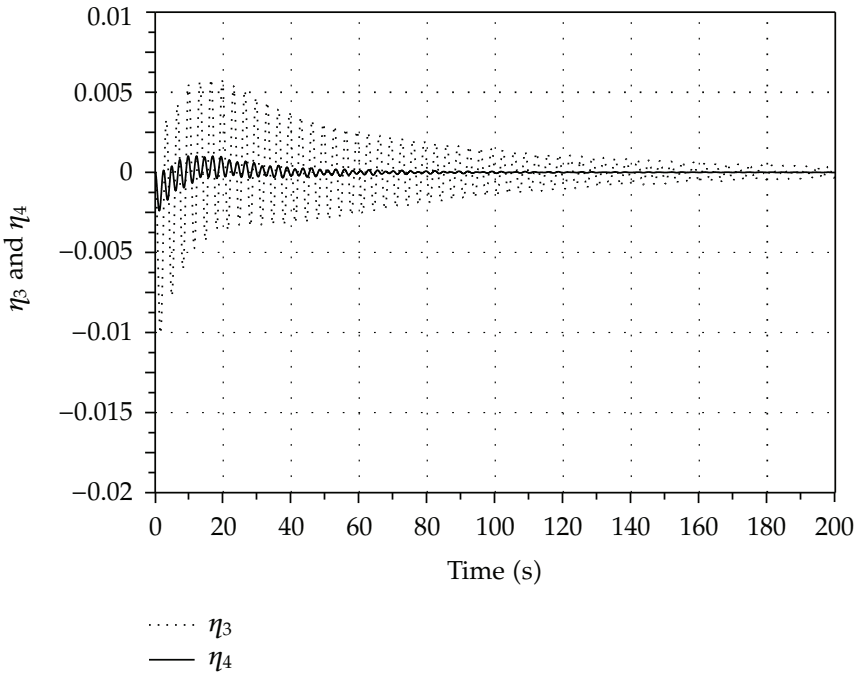

Figure 6: SDRE-based SM controller: modal displacements with external disturbances.

Simulation studies have been performed to test both controllers. Figures 1 and 2 clearly show the performance of the SDRE-based SM controller. The responses of quaternion and angular velocity components reach zero after 70 seconds. Obviously the effect of external disturbances on quaternion and angular velocity is totally removed. From Figure 4 it can be seen that the SDRE-based SM controller stabilizes the closed-loop system of flexible spacecraft and provides quite smooth control torque responses. As shown in Figures 5 and 6 the modal displacements $\left(\mu_{1}-\mu_{1}\right)$ converge to the neighborhood of zero.

On the other hand Figure 7 shows that the optimal Lyapunov-based SM controller provides good trajectories of the quaternions and they reach zero in about 80 seconds. Similarly, from Figure 8 it can be seen that the angular velocities reach zero after 100 seconds. 


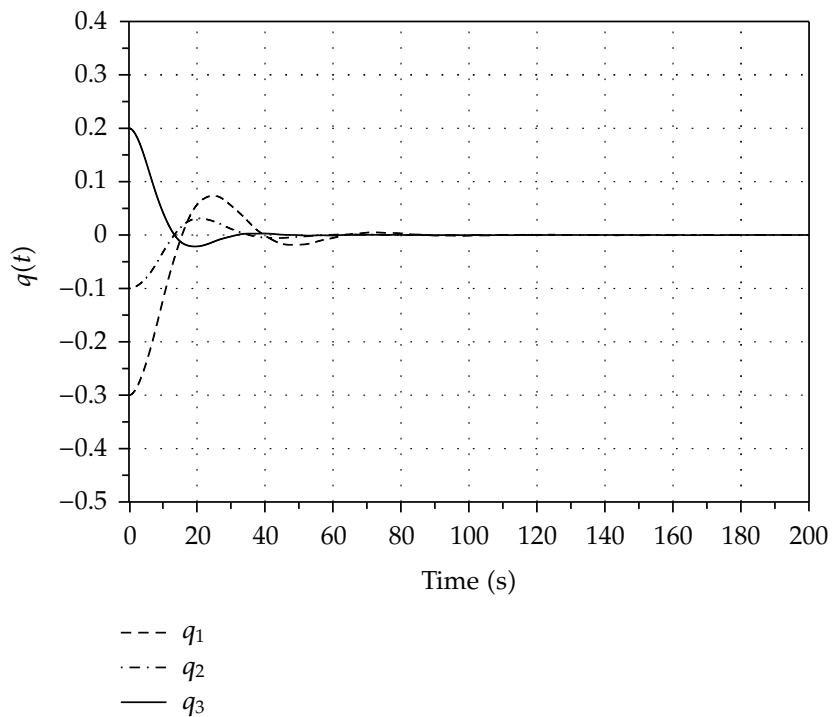

Figure 7: Optimal Lyapunov-based SM controller: components of quaternion vector with external disturbances.

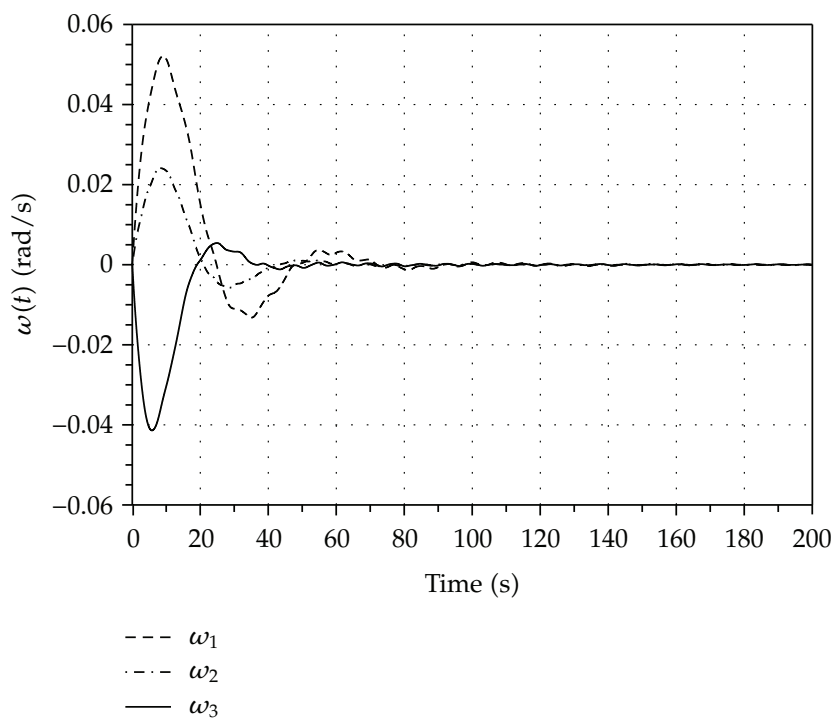

Figure 8: Optimal Lyapunov-based SM controller: components of angular velocity vector with external disturbances.

As shown in Figure 10 the control torques obtained by the optimal Lyapunov-based SM controller are quite smooth although the external disturbances are taken into account. The responses of modal displacements are similar to those obtained by the SDRE-based SM controller (Figures 11 and 12). For both controllers, the sliding vectors are on the sliding manifold $(s=0)$ at time zero and very close to zero thereafter (Figures 3 and 9). 


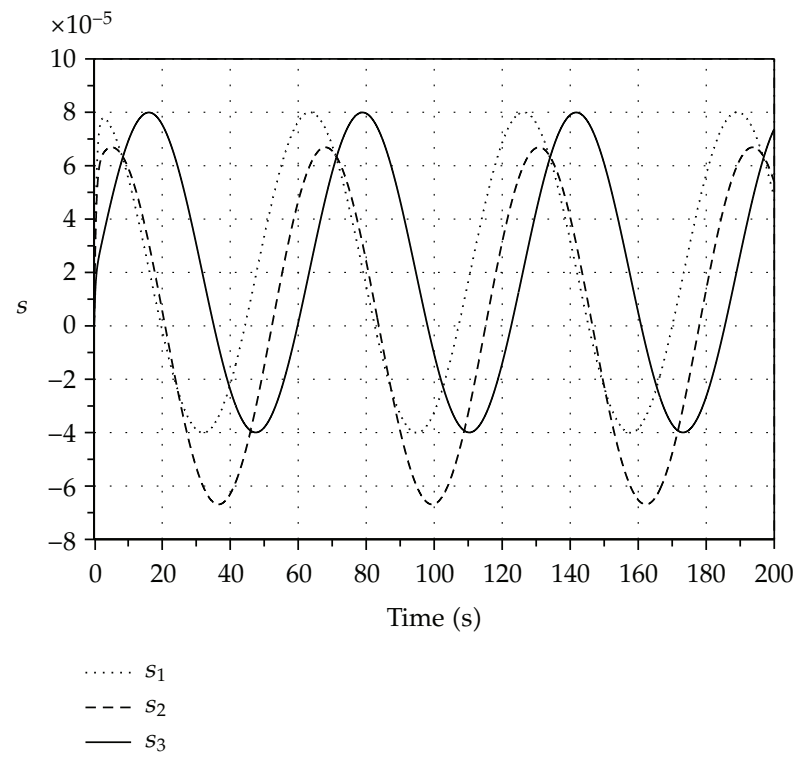

Figure 9: Optimal Lyapunov-based SM controller: switching function with external disturbances.

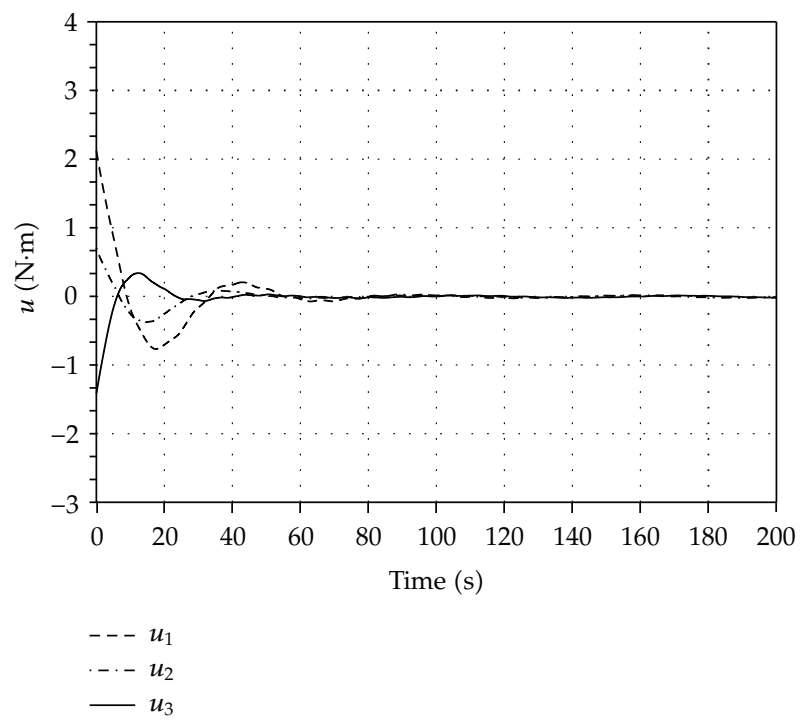

Figure 10: Optimal Lyapunov-based SM controller: control torques with external disturbances.

Making comparisons between the simulation results obtained by the SDRE-based SM controller and optimal Lyapunov-based SM controller, it can be seen that the SDREbased SM controller provides smoother attitude responses and achieves the desired attitude faster. In view of these simulation results, the SDRE-based SM controller seems to be a more useful approach for general cases of attitude regulation problems. Different models and manoeuvres may yield different behaviour. However, the success of the SDRE approach depends on a good choice of the SDC matrix $a(x)$. It is difficult to obtain global stability 


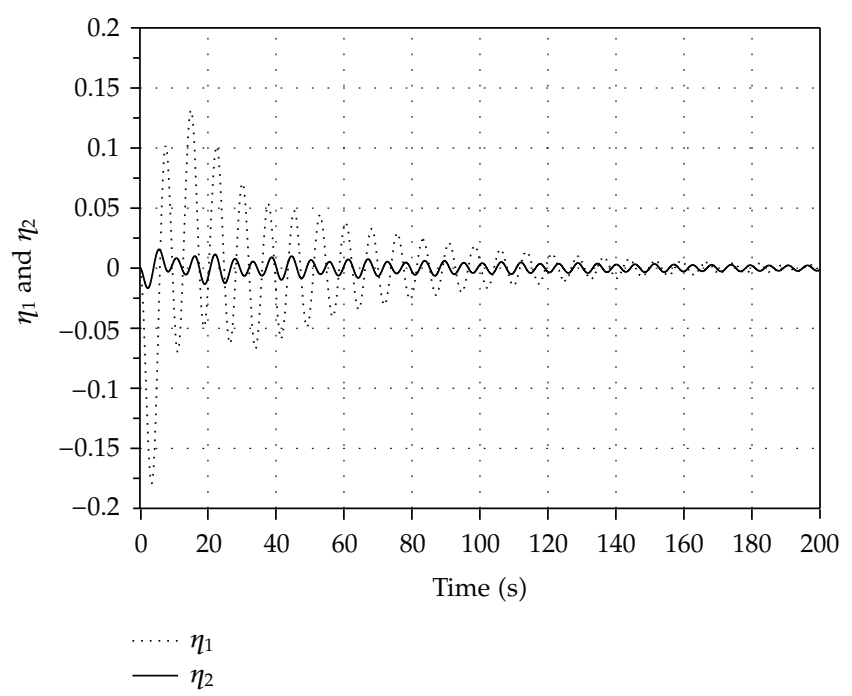

Figure 11: Optimal Lyapunov-based SM controller: modal displacements with external disturbances.

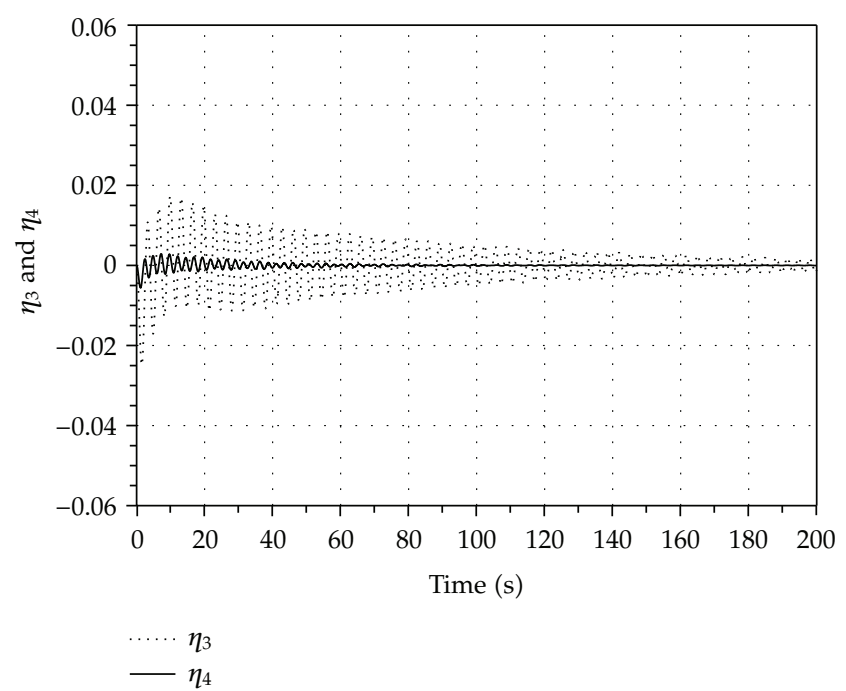

Figure 12: Optimal Lyapunov-based SM controller: modal displacements with external disturbances.

because of the limitations of this technique. On the other hand the difficulty of using the optimal Lyapunov approach is how to find a suitable Lyapunov function. Once this function is known, the optimal Lyapunov approach can be used for the optimal controller design that yields global asymptotic stability.

\section{Conclusion}

We have studied two controller designs for attitude stabilization of a flexible spacecraft. Both the SDRE-based SM controller and optimal Lyapunov-based SM controller have been successfully applied to the spacecraft attitude manoeuvres. To obtain both controller 
designs, ISMC combined with first-order sliding mode and optimal control has been applied to quaternion-based spacecraft attitude manoeuvres with external disturbances and an uncertain inertia matrix. The SDRE and the optimal Lyapunov approaches are used to solve the infinite-time nonlinear optimal control problem. The second method of Lyapunov is used to guarantee the stability of the attitude control system under the action of both controllers. An example of multiaxial attitude manoeuvres is presented and simulation results are given and compared to verify the usefulness of the developed controllers.

\section{Acknowledgment}

The authors thank the referees for their valuable comments that have improved this paper.

\section{References}

[1] L. L. Show and J. C. Juang, "Satellite large angle tracking control design: thruster control approach," in Proceedings of the American Control Conference (ACC '03), pp. 1098-1103, Denver, Colo, USA, June 2003.

[2] R. Sharma and A. Tewari, "Optimal nonlinear tracking of spacecraft attitude maneuvers," IEEE Transactions on Control Systems Technology, vol. 12, no. 5, pp. 677-682, 2004.

[3] W. Luo, Y.-C. Chu, and K.-V. Ling, "Inverse optimal adaptive control for attitude tracking of spacecraft," IEEE Transactions on Automatic Control, vol. 50, no. 11, pp. 1639-1654, 2005.

[4] W. Perruquetti and J. P. Barbot, Sliding Mode Control in Engineering, Marcel Dekker, New York, NY, USA, 2002.

[5] G. Bartolini, A. Ferrara, and E. Usani, "Chattering avoidance by second-order sliding mode control," IEEE Transactions on Automatic Control, vol. 43, no. 2, pp. 241-246, 1998.

[6] B. Yao and M. Tomizuka, "Smooth robust adaptive sliding mode control of manipulators with guaranteed transient performance," in Proceedings of the American Control Conference (ACC '94), vol. 1, pp. 1176-1180, Baltimore, Md, USA, June 1994.

[7] J. S. Park, G. S. Han, H. S. Ahn, and D. H. Kim, "Adaptive approaches on the sliding mode control of robot manipulators," Transactions on Control, Automation and Systems Engineering, vol. 3, no. 1, pp. 15-20, 2001.

[8] H. Xu, M. Mirmirani, and P. Ioannou, "Adaptive sliding mode control design for a hypersonic flight vehicle," Journal of Guidance, Control, and Dynamics, vol. 27, no. 5, pp. 829-838, 2004.

[9] G. Bartolini, A. Pisano, and E. Usai, "Second-order sliding-mode control of container cranes," Automatica, vol. 38, no. 10, pp. 1783-1790, 2002.

[10] A. Damiano, G. L. Gatto, I. Marongiu, and A. Pisano, "Second-order sliding-mode control of DC drives," IEEE Transactions on Industrial Electronics, vol. 51, no. 2, pp. 364-373, 2004.

[11] G. Bartolini, A. Damiano, G. Gatto, I. Marongiu, A. Pisano, and E. Usai, "Robust speed and torque estimation in electrical drives by second-order sliding modes," IEEE Transactions on Control Systems Technology, vol. 11, no. 1, pp. 84-90, 2003.

[12] G. Bartolini, A. Pisano, E. Punta, and E. Usai, "A survey of applications of second-order sliding mode control to mechanical systems," International Journal of Control, vol. 76, no. 9-10, pp. 875-892, 2003.

[13] A. Levant, A. Pridor, R. Gitizadeh, I. Yaesh, and J. Z. Ben-Asher, "Aircraft pitch control via secondorder sliding technique," Journal of Guidance, Control, and Dynamics, vol. 23, no. 4, pp. 586-594, 2000.

[14] C. Pukdeboon, A. S. I. Zinober, and M.-W. L. Thein, "Quasi-continuous higher-order sliding mode controller designs for spacecraft attitude tracking manoeuvres," IEEE Transactions on Industrial Electronics, vol. 57, no. 4, pp. 1436-1444, 2010.

[15] R. Xu, Optimal sliding mode control and stabilization of underactuated systems, dissertation, Electrical Engineering, Ohio State University, 2007.

[16] V. I. Utkin and J. Shi, "Integral sliding mode in systems operating under uncertainty conditions," in Proceedings of the 35th IEEE Conference on Decision and Control, pp. 4591-4596, Kobe, Japan, December 1996. 
[17] C. Pukdeboon and A. S. I. Zinober, "Optimal sliding mode controllers for attitude tracking of spacecraft," in Proceedings of the IEEE Control Applications \& Intelligent Control, pp. 1708-1713, St. Petersburg, Russia, July 2009.

[18] A. Sinha and D. W. Miller, "Optimal sliding mode control of a flexible spacecraft under stochastic disturbances," in Proceedings of the American Control Conference (ACC '03), pp. 188-196, San Francisco, Calif, USA, June 1993.

[19] Q. L. Hu and G. F. Ma, "Optimal sliding mode manoeuvring control and active vibration reduction of flexible spacecraft," Proceedings of the Institution of Mechanical Engineers, Part G: Journal of Aerospace Engineering, vol. 220, no. 4, pp. 317-335, 2006.

[20] S. P. Banks and K. J. Mhana, "Optimal control and stabilization for nonlinear systems," IMA Journal of Mathematical Control and Information, vol. 9, no. 2, pp. 179-196, 1992.

[21] J. R. Cloutier, C. N. D'Souza, and C. P. Mracek, "Nonlinear regulation and nonlinear $H_{\infty}$ control via the state-dependent Riccat equation technique," in Proceedings of the International Conference on Nonlinear Problems in Aviation and Aerospace, Daytona Beach, Fla, USA, May 1996.

[22] D. K. Parrish and D. B. Ridgely, "Attitude control of a satellite using the SDRE method," in Proceedings of the American Control Conference (ACC '07), pp. 942-946, Albuquerque, NM, USA, June 1997.

[23] D. T. Stansberg and J. R. Cloutier, "Position and attitude control of a spacecraft using the statedependent Riccati equation techniques," in Proceedings of the American Control Conference (ACC '00), Chicago, Ill, USA, June 2000.

[24] Y. Zhang, S. K. Agrawal, P. R. Hemanshu, and M. J. Piovoso, "Optimal control using state dependent Riccati equation (SDRE) for a flexible cable transporter system with arbitrarily varying lengths," in Proceedings of the IEEE Conference on Control Applications, Toronto, Canada, August 2005.

[25] R. Ortega, A. Loria, P. J. Nicklasson, and H. J. Sira-Ramirez, Passivity-Based Control of Euler-Lagrange Systems: Mechanical, Electrical and Electromechanical Applications, Springer, London, UK, 1998.

[26] A. Loria, R. Kelly, R. Ortega, and V. Santibanez, "On global output feedback regulation of EulerLagrange systems with bounded inputs," IEEE Transactions on Automatic Control, vol. 42, no. 8, pp. 1138-1143, 1997.

[27] G. Escobar, R. Ortega, and H. Sira-Ramírez, "Output-feedback global stabilization of a nonlinear benchmark system using a saturated passivity-based controller," IEEE Transactions on Control Systems Technology, vol. 7, no. 2, pp. 289-293, 1999.

[28] A. El-Gohary, "Optimal control of an angular motion of a rigid body during infinite and finite time intervals," Applied Mathematics and Computation, vol. 141, no. 2-3, pp. 541-551, 2003.

[29] A. El-Gohary, "Optimal control of a programmed motion of a rigid spacecraft using redundant kinematics parameterizations," Chaos, Solitons E Fractals, vol. 26, no. 4, pp. 1053-1063, 2005.

[30] A. El-Gohary, "Optimal control for the attitude stabilization of a rigid body using non-redundant parameters," International Journal of Mechanical Sciences, vol. 48, no. 9, pp. 1004-1013, 2006.

[31] A. El-Gohary and S. Tawfik, "Optimal control of a rigid body motion using Euler parameters without angular velocity measurements," Mechanics Research Communications, vol. 37, no. 3, pp. 354-359, 2010.

[32] S. P. Banks and S. K. Al-Jurani, "Pseudo-linear systems, Lie algebras and stability," Research Report 529, University of Sheffield, 1994.

[33] S. Di Gennaro, "Output stabilization of flexible spacecraft with active vibration suppression," IEEE Transactions on Aerospace and Electronic Systems, vol. 39, no. 3, pp. 747-759, 2003.

[34] J. R. Wertz, Spacecraft Attitude Determination and Control, Kluwer Academic, Dordrecht, London, UK, 1978.

[35] M. D. Shuster, "A survey of attitude representations," Journal of the Astronautical Sciences, vol. 41, no. 4, pp. 439-517, 1993.

[36] T. Cimen, "State-dependent Riccati equation (SDRE) control: a survey," in Proceedings of the 17th International Federation of Automatic Control World Congress (IFAC '08), Seoul, Korea, July 2008.

[37] N. N. Krasovskii, "On the stabilization of unstable motions by additional forces when the feedback loop is incomplete," Journal of Applied Mathematics and Mechanics, vol. 27, no. 1963, pp. 971-1004, 1963. 


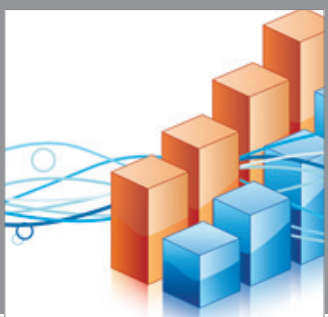

Advances in

Operations Research

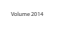

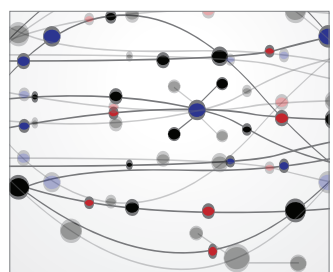

\section{The Scientific} World Journal
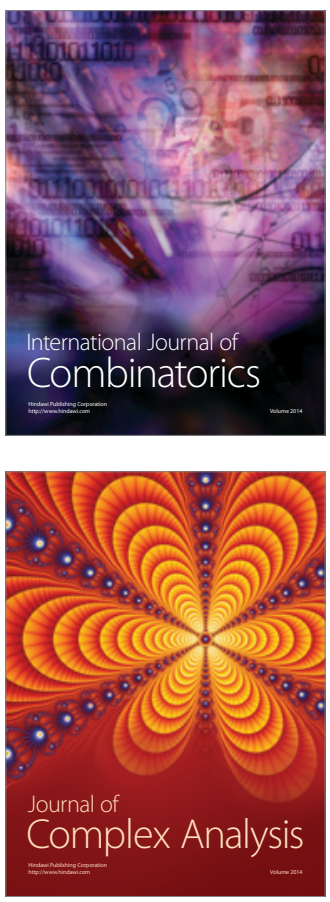

International Journal of

Mathematics and

Mathematical

Sciences
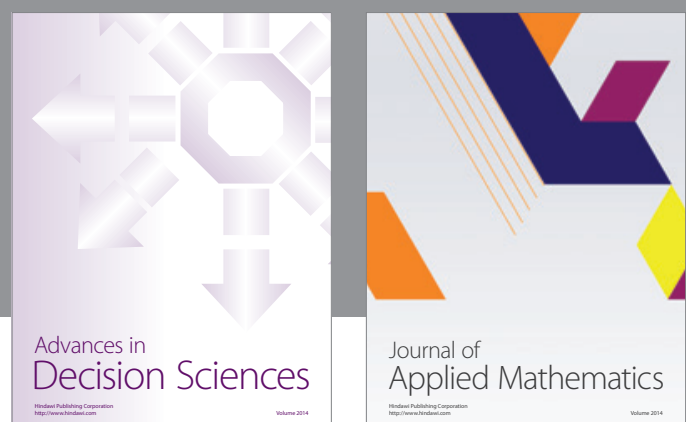

Journal of

Applied Mathematics
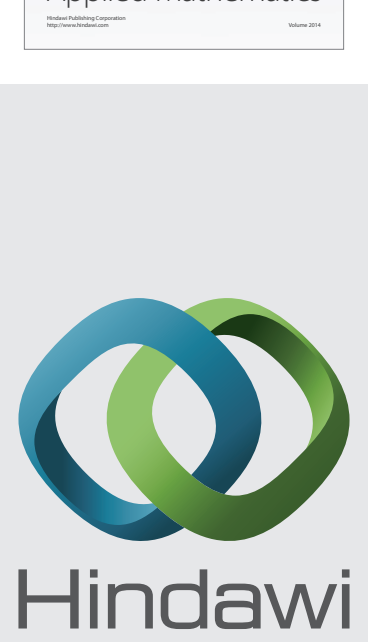

Submit your manuscripts at http://www.hindawi.com
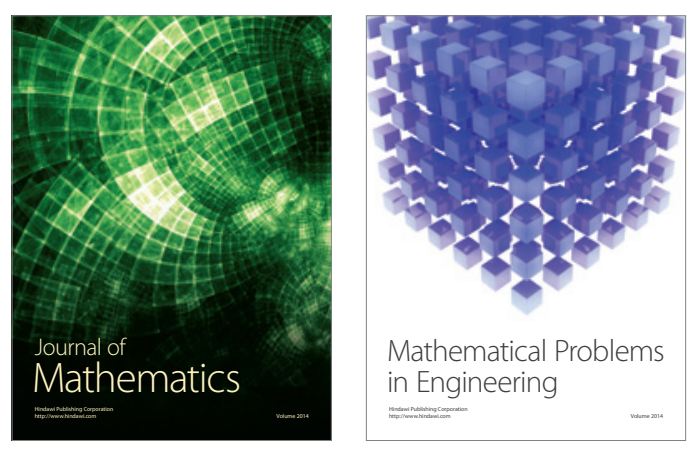

Mathematical Problems in Engineering
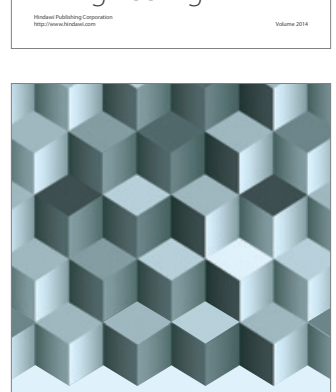

Journal of

Function Spaces
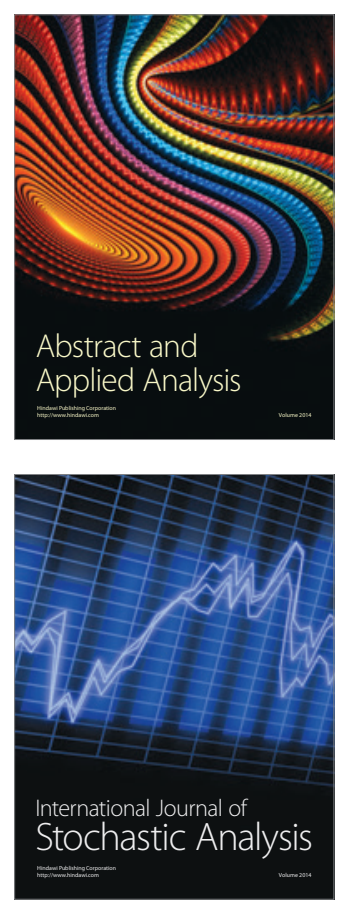

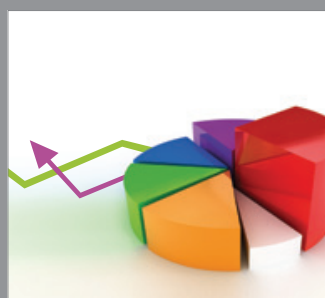

ournal of

Probability and Statistics

Promensencen
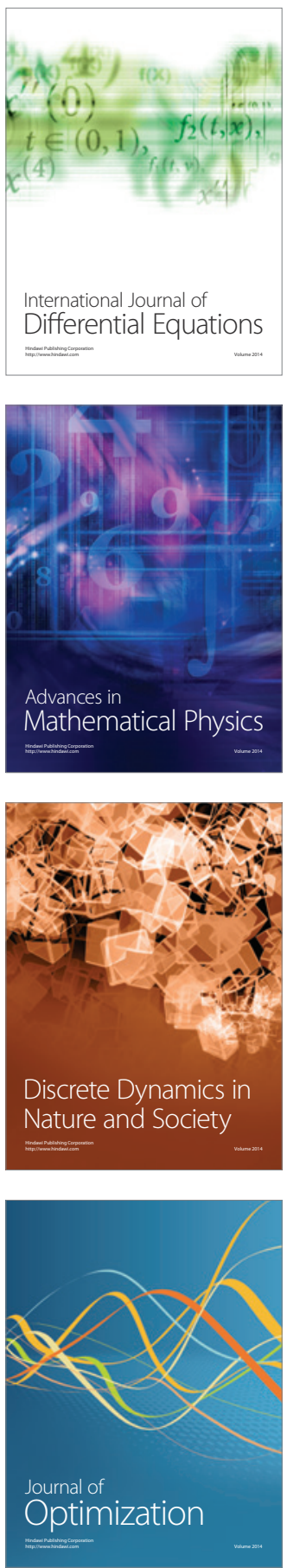